\title{
A Nitsche-based method for unilateral contact problems: numerical analysis
}

\author{
Franz Chouly * $\quad$ Patrick Hild ${ }^{\dagger}$
}

\begin{abstract}
We introduce a Nitsche-based formulation for the finite element discretization of the unilateral contact problem in linear elasticity. It features a weak treatment of the non-linear contact conditions through a consistent penalty term. Without any additional assumption on the contact set, we can prove theoretically its fully optimal convergence rate in the $H^{1}(\Omega)$ norm for linear finite elements in two dimensions, which is $O\left(h^{\frac{1}{2}+\nu}\right)$ when the solution lies in $H^{\frac{3}{2}+\nu}(\Omega), 0<\nu \leq 1 / 2$. An interest of the formulation is that, conversely to Lagrange multiplier-based methods, no other unknown is introduced and no discrete inf-sup condition needs to be satisfied.
\end{abstract}

Key words: unilateral contact, finite elements, Nitsche's method.

AMS Subject Classification: 65N12, 65N30, 74M15.

\section{Introduction}

The numerical implementation of contact and impact problems in solid mechanics generally uses the Finite Element Method (FEM) (see [17, 18, 20, 27, 29, 36, 38]). These problems involve non-linear contact conditions on a part of the boundary that lead naturally to a variational inequality (see, e.g., [14]). In this paper, we limit ourselves to the case of frictionless unilateral contact in plane linear elasticity. Indeed, this case contains the key difficulties related to the non-linear contact conditions. We also limit our study to the approximation with continuous piecewise linear finite elements, which is the most standard approach.

We introduce a special FEM inspired from Nitsche method [31, 2, 35, 4, 21]. Conversely to standard penalization techniques [27], the resulting method is consistent. Moreover, unlike mixed methods (see, e.g., [20]), no additional unknown (Lagrange multiplier) is needed. However, the most interesting in this method is that we are able to prove its optimal convergence in the $H^{1}(\Omega)$-norm of order $O\left(h^{\frac{1}{2}+\nu}\right)$ provided the solution has a regularity $H^{\frac{3}{2}+\nu}(\Omega), 0<\nu \leq 1 / 2$.

\footnotetext{
*Laboratoire de Mathématiques - UMR CNRS 6623, Université de Franche Comté, 16 route de Gray, 25030 Besançon Cedex, France. email: franz.chouly@univ-fcomte.fr

${ }^{\dagger}$ Institut de Mathématiques de Toulouse - UMR CNRS 5219, Université Paul Sabatier, 118 route de Narbonne, 31062 Toulouse Cedex 9, France. email: patrick.hild@math.univ-toulouse.fr
} 
Furthermore, we do not need any additional assumption on the contact zone, such as an increased regularity of the contact stress or a finite number of transitions between contact and non-contact. Besides, the standard FEM for contact consists in a direct conforming approximation of the variational inequality, with the elastic displacement as the only unknown. For this standard FEM and also for all the other approaches such as mixed/hybrid methods (e.g., [7]), stabilized mixed methods (e.g., [23]), penalty methods (e.g., [10]), no such proof of optimal convergence has been established to the best of our knowledge in the case the solution $\mathbf{u}$ is in $H^{\frac{3}{2}+\nu}(\Omega)$ $(0<\nu \leq 1 / 2)$. The first analyzes in $[34,19,20]$ were sub-optimal with a convergence in $O\left(h^{\frac{1}{2}+\frac{\nu}{2}}\right)$ and these results were recently improved in [24] to obtain a convergence rate of $O\left(h^{\frac{1}{2}+\frac{\nu}{2}+\nu^{2}}\right)$ when $(0<\nu<1 / 2)$, of $O(h \sqrt{|\ln (h)|})$ when $\nu=1 / 2$ and in [33] where a convergence of $O(h)$ is obtained when $\nu=1 / 2+\varepsilon(\varepsilon>0$ arbitrarily small). In fact, additional assumptions on the finiteness of transition points between contact and non-contact are needed to recover optimality (see [6] for the case $0<\nu<1 / 2$ and [25] for the case $\nu=1 / 2$ in the case of the standard FEM). Our paper is outlined as follows. In Section 2, we recall the continuous (strong and weak) formulations for unilateral contact problems and introduce our Nitsche-based FEM. In Section 3 , we carry out the numerical analysis of this method: we prove its consistency, the existence and uniqueness of solutions, and at least its optimal convergence. Conclusion and perspectives are drawn in Section 4.

Let us introduce some useful notations. In what follows, bold letters like $\mathbf{u}, \mathbf{v}$, indicate vector or tensor valued quantities, while the capital ones (e.g., V, K ...) represent functional sets involving vector fields. As usual, we denote by $\left(H^{s}(.)\right)^{d}, s \in \mathbb{R}, d=1,2$ the Sobolev spaces in one or two space dimensions (see [1]). The usual norm of $\left(H^{s}(D)\right)^{d}$ is denoted by $\|\cdot\|_{s, D}$ and we keep the same notation when $d=1$ or $d=2$. The letter $C$ stands for a generic constant, independent of the discretization parameters.

\section{Setting}

\subsection{The unilateral contact problem}

We consider an elastic body $\Omega$ in $\mathbb{R}^{2}$ where plane small strain assumptions are made. The boundary $\partial \Omega$ of $\Omega$ is polygonal and we suppose that $\partial \Omega$ consists in three nonoverlapping parts $\Gamma_{D}, \Gamma_{N}$ and the contact boundary $\Gamma_{C}$, with meas $\left(\Gamma_{D}\right)>0$ and meas $\left(\Gamma_{C}\right)>0$. The contact boundary is supposed to be a straight line segment to simplify ; the normal unit outward vector on $\partial \Omega$ is denoted $\mathbf{n}$. In its initial stage, the body is in contact on $\Gamma_{C}$ with a rigid foundation (the extension to two elastic bodies in contact can be easily made, at least for small strain models) and we suppose that the unknown final contact zone after deformation will be included into $\Gamma_{C}$. The body is clamped on $\Gamma_{D}$ for the sake of simplicity. It is subjected to volume forces $\mathbf{f} \in\left(L^{2}(\Omega)\right)^{2}$ and to surface loads $\mathbf{g} \in\left(L^{2}\left(\Gamma_{N}\right)\right)^{2}$.

The unilateral contact problem in linear elasticity consists in finding the displacement field 
$\mathbf{u}: \Omega \rightarrow \mathbb{R}^{2}$ verifying the equations and conditions (1)-(2):

$$
\begin{aligned}
\operatorname{div} \boldsymbol{\sigma}(\mathbf{u})+\mathbf{f} & =\mathbf{0} & & \text { in } \Omega, \\
\boldsymbol{\sigma}(\mathbf{u}) & =\mathbf{A} \boldsymbol{\varepsilon}(\mathbf{u}) & & \text { in } \Omega, \\
\mathbf{u} & =\mathbf{0} & & \text { on } \Gamma_{D} \\
\boldsymbol{\sigma}(\mathbf{u}) \mathbf{n} & =\mathbf{g} & & \text { on } \Gamma_{N}
\end{aligned}
$$

where $\boldsymbol{\sigma}=\left(\sigma_{i j}\right), 1 \leq i, j \leq 2$, stands for the stress tensor field and div denotes the divergence operator of tensor valued functions. The notation $\varepsilon(\mathbf{v})=\left(\boldsymbol{\nabla} \mathbf{v}+\boldsymbol{\nabla} \mathbf{v}^{T}\right) / 2$ represents the linearized strain tensor field and $\mathbf{A}$ is the fourth order symmetric elasticity tensor having the usual uniform ellipticity and boundedness property. For any displacement field $\mathbf{v}$ and for any density of surface forces $\boldsymbol{\sigma}(\mathbf{v}) \mathbf{n}$ defined on $\partial \Omega$ we adopt the following notation

$$
\mathbf{v}=v_{n} \mathbf{n}+v_{\mathbf{t}} \quad \text { and } \quad \boldsymbol{\sigma}(\mathbf{v}) \mathbf{n}=\sigma_{n}(\mathbf{v}) \mathbf{n}+\sigma_{\mathbf{t}}(\mathbf{v}),
$$

where $v_{\mathbf{t}}\left(\right.$ resp. $\left.\sigma_{\mathbf{t}}(\mathbf{v})\right)$ are the tangential components of $\mathbf{v}($ resp. $\boldsymbol{\sigma}(\mathbf{v}) \mathbf{n})$. The conditions describing unilateral contact without friction on $\Gamma_{C}$ are:

$$
\begin{aligned}
u_{n} \leq 0 & (i) \\
\sigma_{n}(\mathbf{u}) \leq 0 & (i i) \\
\sigma_{n}(\mathbf{u}) u_{n}=0 & (i i i) \\
\sigma_{\mathbf{t}}(\mathbf{u})=0 & (i v)
\end{aligned}
$$

We introduce the Hilbert space $\mathbf{V}$ and the convex cone $\mathbf{K}$ of admissible displacements which satisfy the noninterpenetration on the contact zone $\Gamma_{C}$ :

$$
\mathbf{V}:=\left\{\mathbf{v} \in\left(H^{1}(\Omega)\right)^{2}: \mathbf{v}=\mathbf{0} \text { on } \Gamma_{D}\right\}, \quad \mathbf{K}:=\left\{\mathbf{v} \in \mathbf{V}: v_{n}=\mathbf{v} \cdot \mathbf{n} \leq 0 \text { on } \Gamma_{C}\right\} .
$$

Define

$$
a(\mathbf{u}, \mathbf{v}):=\int_{\Omega} \boldsymbol{\sigma}(\mathbf{u}): \varepsilon(\mathbf{v}) d \Omega, \quad L(\mathbf{v}):=\int_{\Omega} \mathbf{f} \cdot \mathbf{v} d \Omega+\int_{\Gamma_{N}} \mathbf{g} \cdot \mathbf{v} d \Gamma
$$

for any $\mathbf{u}$ and $\mathbf{v}$ in $\mathbf{V}$. From the previous assumptions, we know that $a(\cdot, \cdot)$ is bilinear, symmetric, $\mathbf{V}$-elliptic and continuous on $\mathbf{V} \times \mathbf{V}$. We know also that $L(\cdot)$ is a continuous linear form on $\mathbf{V}$. The weak formulation of Problem (1)-(2), as a variational inequality (see [14, 20, 27]), is:

$$
\left\{\begin{array}{l}
\text { Find } \mathbf{u} \in \mathbf{K} \text { such that: } \\
a(\mathbf{u}, \mathbf{v}-\mathbf{u}) \geq L(\mathbf{v}-\mathbf{u}), \quad \forall \mathbf{v} \in \mathbf{K} .
\end{array}\right.
$$

Stampacchia's Theorem ensures that Problem (3) admits a unique solution. 


\subsection{The Nitsche-based finite element method}

Let $\mathbf{V}^{h} \subset \mathbf{V}$ be a family of finite dimensional vector spaces (see [11]) indexed by $h$ coming from a family $\mathcal{T}^{h}$ of triangulations of the domain $\Omega\left(h=\max _{T \in \mathcal{T}^{h}} h_{T}\right.$ where $h_{T}$ is the diameter of $T$ ). The family of triangulations is supposed regular, i.e., there exists $\sigma>0$ such that $\forall T \in \mathcal{T}^{h}, h_{T} / \rho_{T} \leq \sigma$ where $\rho_{T}$ denotes the radius of the inscribed circle in $T$. We choose standard continuous and piecewise affine functions, i.e.:

$$
\mathbf{V}^{h}=\left\{\mathbf{v}^{h} \in(C(\bar{\Omega}))^{2}: \mathbf{v}_{\left.\right|_{T}} \in\left(P_{1}(T)\right)^{2}, \forall T \in \mathcal{T}^{h}, \mathbf{v}^{h}=\mathbf{0} \text { on } \Gamma_{D}\right\}
$$

Let us introduce the notation $[\cdot]_{+}$for the positive part of a scalar quantity $a \in \mathbb{R}$ :

$$
[a]_{+}=\left\{\begin{array}{rr}
a & \text { if } a>0 \\
0 & \text { otherwise }
\end{array}\right.
$$

In the rest of this paper, we will make an extensive use of the following properties:

$$
a \leq[a]_{+}, \quad a[a]_{+}=[a]_{+}^{2}, \quad \forall a \in \mathbb{R} .
$$

Note that conditions (4) can be straightforwardly extended to real valued functions. The derivation of a Nitsche-based method comes from the observation that the contact conditions (2) can be reformulated as follows:

Proposition 2.1. Let $\gamma>0$. The contact conditions (2) (i)-(iii) on $\Gamma_{C}$ are equivalent to:

$$
\sigma_{n}(\mathbf{u})=-\frac{1}{\gamma}\left[u_{n}-\gamma \sigma_{n}(\mathbf{u})\right]_{+} \cdot
$$

Proof: Let $\mathbf{u}$ be a regular vector field on $\Omega$ such that (2) (i)-(iii) holds. The condition (2)-(ii) yields either $\sigma_{n}(\mathbf{u})<0$ or $\sigma_{n}(\mathbf{u})=0$. Suppose first that $\sigma_{n}(\mathbf{u})<0$. Then (2)-(iii) implies that $u_{n}=0$. In this case, it holds:

$$
-\frac{1}{\gamma}\left[u_{n}-\gamma \sigma_{n}(\mathbf{u})\right]_{+}=-\frac{1}{\gamma}\left[-\gamma \sigma_{n}(\mathbf{u})\right]_{+}=\sigma_{n}(\mathbf{u}) .
$$

Then, suppose that $\sigma_{n}(\mathbf{u})=0$. The condition (2)-(i) can also be expressed as $\left[u_{n}\right]_{+}=0$, so:

$$
-\frac{1}{\gamma}\left[u_{n}-\gamma \sigma_{n}(\mathbf{u})\right]_{+}=-\frac{1}{\gamma}\left[u_{n}\right]_{+}=0=\sigma_{n}(\mathbf{u}) .
$$

Reciprocally, let $\mathbf{u}$ such that (5) holds. Note first that it implies $\sigma_{n}(\mathbf{u}) \leq 0$, so that (2)-(ii) holds. Take first the case $\sigma_{n}(\mathbf{u})=0$, then (5) can be rewritten as:

$$
0=-\frac{1}{\gamma}\left[u_{n}\right]_{+}
$$

which is equivalent to condition (2)-(i). Since $\sigma_{n}(\mathbf{u})=0$, then condition (2)-(iii) $\left(\sigma_{n}(\mathbf{u}) u_{n}=0\right)$ also holds. 
We now consider the case $\sigma_{n}(\mathbf{u})<0$. From $(5),\left[u_{n}-\gamma \sigma_{n}(\mathbf{u})\right]_{+}>0$, so that in this case

$$
-\gamma \sigma_{n}(\mathbf{u})=\left[u_{n}-\gamma \sigma_{n}(\mathbf{u})\right]_{+}=u_{n}-\gamma \sigma_{n}(\mathbf{u}),
$$

from which comes $u_{n}=0$, so that both (2) (i) and (iii) hold.

Remark 2.2. Note that condition (5) is still equivalent to (2) (i)-(iii) on $\Gamma_{C}$ when $\gamma$ is a positive function defined on $\Gamma_{C}$ instead of a positive constant.

Let now $\mathbf{u}$ be the solution of the unilateral contact problem in its strong form (1)-(2), sufficiently regular so that all the following calculations make sense. From the Green formula and equations (1), (2)-(iv), it holds for every $\mathbf{v} \in \mathbf{V}$ :

$$
a(\mathbf{u}, \mathbf{v})-\int_{\Gamma_{C}} \sigma_{n}(\mathbf{u}) v_{n} d \Gamma=L(\mathbf{v})
$$

Note that $v_{n}=\left(v_{n}-\gamma \sigma_{n}(\mathbf{v})\right)+\gamma \sigma_{n}(\mathbf{v})$, so that:

$$
a(\mathbf{u}, \mathbf{v})-\int_{\Gamma_{C}} \gamma \sigma_{n}(\mathbf{u}) \sigma_{n}(\mathbf{v}) d \Gamma-\int_{\Gamma_{C}} \sigma_{n}(\mathbf{u})\left(v_{n}-\gamma \sigma_{n}(\mathbf{v})\right) d \Gamma=L(\mathbf{v}) .
$$

Finally, using condition (5), we obtain:

$$
a(\mathbf{u}, \mathbf{v})-\int_{\Gamma_{C}} \gamma \sigma_{n}(\mathbf{u}) \sigma_{n}(\mathbf{v}) d \Gamma+\int_{\Gamma_{C}} \frac{1}{\gamma}\left[u_{n}-\gamma \sigma_{n}(\mathbf{u})\right]_{+}\left(v_{n}-\gamma \sigma_{n}(\mathbf{v})\right) d \Gamma=L(\mathbf{v}) .
$$

Formula (6) is the starting point of our Nitsche-based formulation. Remark that it may have no sense at the continuous level if $\mathbf{u}$ lacks of regularity $(\mathbf{u} \in \mathbf{V}$ only is not sufficient for instance to justify the above calculations). Nevertheless, and as in the stabilized Lagrange multiplier method [23], we consider in what follows that $\gamma$ is a positive piecewise constant function on the contact interface $\Gamma_{C}$ : for any $x \in \Gamma_{C}$, let $T$ be an element such that $x \in T$ and set:

$$
\gamma(x)=\gamma_{0} h_{T}
$$

where $\gamma_{0}$ is a positive constant. This allows to define a discrete counterpart of (6). Let us introduce for this purpose the discrete linear operator

$$
P_{\gamma}: \begin{array}{llc}
\mathbf{V}^{h} & \rightarrow L^{2}\left(\Gamma_{C}\right) \\
\mathbf{v}^{h} & \mapsto v_{n}^{h}-\gamma \sigma_{n}\left(\mathbf{v}^{h}\right)
\end{array}
$$

and also the bilinear form:

$$
A_{\gamma}\left(\mathbf{u}^{h}, \mathbf{v}^{h}\right)=a\left(\mathbf{u}^{h}, \mathbf{v}^{h}\right)-\int_{\Gamma_{C}} \gamma \sigma_{n}\left(\mathbf{u}^{h}\right) \sigma_{n}\left(\mathbf{v}^{h}\right) d \Gamma
$$


Remark 2.3. With the previous notations, we see that problem (3) could be formally written as follows :

$$
\left\{\begin{array}{l}
\text { Find a sufficiently regular } \mathbf{u} \in \mathbf{V} \text { such that: } \\
A_{\gamma}(\mathbf{u}, \mathbf{v})+\int_{\Gamma_{C}} \frac{1}{\gamma}\left[P_{\gamma}(\mathbf{u})\right]_{+} P_{\gamma}(\mathbf{v}) d \Gamma=L(\mathbf{v}), \text { for all sufficiently regular } \mathbf{v} \in \mathbf{V}
\end{array}\right.
$$

Our Nitsche-based method then reads:

$$
\left\{\begin{array}{l}
\text { Find } \mathbf{u}^{h} \in \mathbf{V}^{h} \text { such that: } \\
A_{\gamma}\left(\mathbf{u}^{h}, \mathbf{v}^{h}\right)+\int_{\Gamma_{C}} \frac{1}{\gamma}\left[P_{\gamma}\left(\mathbf{u}^{h}\right)\right]_{+} P_{\gamma}\left(\mathbf{v}^{h}\right) d \Gamma=L\left(\mathbf{v}^{h}\right), \quad \forall \mathbf{v}^{h} \in \mathbf{V}^{h}
\end{array}\right.
$$

\subsection{A reinterpretation of the method}

The aim of this subsection is to show some links between the proposed method and the stabilized mixed/hybrid finite element methods (which do not require inf-sup conditions) introduced in [26]. We define the auxiliary variable

$$
\lambda^{h}:=-\frac{1}{\gamma}\left[P_{\gamma}\left(\mathbf{u}^{h}\right)\right]_{+},
$$

which can be interpreted as the $L^{2}$-projection of the quantity $-\frac{1}{\gamma} P_{\gamma}\left(\mathbf{u}^{h}\right)$ on the convex cone:

$$
L_{-}^{2}\left(\Gamma_{C}\right):=\left\{\mu \in L^{2}\left(\Gamma_{C}\right) \mid \mu \leq 0 \text { a.e. on } \Gamma_{C}\right\} .
$$

This means in particular that $\lambda^{h} \in L_{-}^{2}\left(\Gamma_{C}\right)$ verifies the following inequality, for all $\mu \in L_{-}^{2}\left(\Gamma_{C}\right)$ :

$$
\int_{\Gamma_{C}}\left(\mu-\lambda^{h}\right)\left(-\frac{1}{\gamma} P_{\gamma}\left(\mathbf{u}^{h}\right)-\lambda^{h}\right) d \Gamma \leq 0
$$

that is the standard characterization of the projection onto a closed convex set. We then use the definition of the operator $P_{\gamma}$ to obtain

$$
\begin{aligned}
\int_{\Gamma_{C}}\left(\mu-\lambda^{h}\right)\left(-\frac{1}{\gamma} P_{\gamma}\left(\mathbf{u}^{h}\right)-\lambda^{h}\right) d \Gamma & =\int_{\Gamma_{C}}\left(\mu-\lambda^{h}\right)\left(-\frac{1}{\gamma} u_{n}^{h}+\sigma_{n}\left(\mathbf{u}^{h}\right)-\lambda^{h}\right) d \Gamma \\
& =-\int_{\Gamma_{C}} \frac{1}{\gamma}\left(\left(\mu-\lambda^{h}\right) u_{n}^{h}+\gamma\left(\mu-\lambda^{h}\right)\left(\lambda^{h}-\sigma_{n}\left(\mathbf{u}^{h}\right)\right)\right) d \Gamma .
\end{aligned}
$$

Since $\gamma$ is a positive piecewise constant function on $\Gamma_{C}$ (see $\left.(7)\right)$, the above equalities prove that the inequality (9) is equivalent to:

$$
\int_{\Gamma_{C}}\left(\mu-\lambda^{h}\right) u_{n}^{h} d \Gamma+\int_{\Gamma_{C}} \gamma\left(\mu-\lambda^{h}\right)\left(\lambda^{h}-\sigma_{n}\left(\mathbf{u}^{h}\right)\right) d \Gamma \geq 0
$$


The next step is to rewrite the left hand-side of the Nitsche-based method (8) as follows:

$$
\begin{aligned}
& A_{\gamma}\left(\mathbf{u}^{h}, \mathbf{v}^{h}\right)+\int_{\Gamma_{C}} \frac{1}{\gamma}\left[P_{\gamma}\left(\mathbf{u}^{h}\right)\right]_{+} P_{\gamma}\left(\mathbf{v}^{h}\right) d \Gamma \\
= & a\left(\mathbf{u}^{h}, \mathbf{v}^{h}\right)-\int_{\Gamma_{C}} \gamma \sigma_{n}\left(\mathbf{u}^{h}\right) \sigma_{n}\left(\mathbf{v}^{h}\right) d \Gamma-\int_{\Gamma_{C}} \lambda^{h}\left(v_{n}^{h}-\gamma \sigma_{n}\left(\mathbf{v}^{h}\right)\right) d \Gamma \\
= & a\left(\mathbf{u}^{h}, \mathbf{v}^{h}\right)-\int_{\Gamma_{C}} \lambda^{h} v_{n}^{h} d \Gamma+\int_{\Gamma_{C}} \gamma\left(\lambda^{h}-\sigma_{n}\left(\mathbf{u}^{h}\right)\right) \sigma_{n}\left(\mathbf{v}^{h}\right) d \Gamma .
\end{aligned}
$$

We combine this last result to the inequality (10) to obtain an equivalent formulation of the Nitsche-based method:

$$
\left\{\begin{array}{l}
\text { Find }\left(\mathbf{u}^{h}, \lambda^{h}\right) \in \mathbf{V}^{h} \times L_{-}^{2}\left(\Gamma_{C}\right) \text { such that: } \\
a\left(\mathbf{u}^{h}, \mathbf{v}^{h}\right)-\int_{\Gamma_{C}} \lambda^{h} v_{n}^{h} d \Gamma+\int_{\Gamma_{C}} \gamma\left(\lambda^{h}-\sigma_{n}\left(\mathbf{u}^{h}\right)\right) \sigma_{n}\left(\mathbf{v}^{h}\right) d \Gamma=L\left(\mathbf{v}^{h}\right), \quad \forall \mathbf{v}^{h} \in \mathbf{V}^{h}, \\
\int_{\Gamma_{C}}\left(\mu-\lambda^{h}\right) u_{n}^{h} d \Gamma+\int_{\Gamma_{C}} \gamma\left(\mu-\lambda^{h}\right)\left(\lambda^{h}-\sigma_{n}\left(\mathbf{u}^{h}\right)\right) d \Gamma \geq 0, \quad \forall \mu \in L_{-}^{2}\left(\Gamma_{C}\right) .
\end{array}\right.
$$

We finally observe that the Nitsche-based method can be regarded as a mixed method with a stabilization term (see [3]). This makes it close to the stabilized method proposed and analyzed in [23], the difference being that in our case, the space for the Lagrange multiplier $\lambda^{h}$ is $L_{-}^{2}\left(\Gamma_{C}\right)$. A similar analogy has already been noticed in [35] in the case of elliptic problems with Dirichlet boundary conditions.

\section{Analysis of the Nitsche-based method}

In this section, we carry out the mathematical analysis of the method (8). A difference between Nitsche and classical penalty methods [27] is that this former is consistent, which we first show in $§ 3.1$. The proof of well-posedness of the (non-linear) discrete problem (8) is carried out in $\S 3.2$. The error analysis is finally detailed in $\S 3.3$. We show that the method converges optimally for a fixed value of $\gamma_{0}$ and when the mesh size $h$ vanishes.

\subsection{Consistency}

As well as the Nitsche's method for second order elliptic problems with Dirichlet boundary condition or domain decomposition [4], our Nitsche-based formulation (8) for unilateral contact is consistent:

Lemma 3.1. The Nitsche-based method for contact is consistent: suppose that the solution $\mathbf{u}$ of (1)-(2) is in $\left(H^{\frac{3}{2}+\nu}(\Omega)\right)^{2}$, with $\nu>0$, then $\mathbf{u}$ is also solution of

$$
A_{\gamma}\left(\mathbf{u}, \mathbf{v}^{h}\right)+\int_{\Gamma_{C}} \frac{1}{\gamma}\left[P_{\gamma}(\mathbf{u})\right]_{+} P_{\gamma}\left(\mathbf{v}^{h}\right) d \Gamma=L\left(\mathbf{v}^{h}\right), \quad \forall \mathbf{v}^{h} \in \mathbf{V}^{h}
$$


Proof: Let $\mathbf{u}$ be the solution of $(1)-(2)$ and take $\mathbf{v}^{h} \in \mathbf{V}^{h}$. Since $\mathbf{u} \in\left(H^{\frac{3}{2}+\nu}(\Omega)\right)^{2}$ and $\nu>0$, we have $\sigma_{n}(\mathbf{u}) \in H^{\nu}\left(\Gamma_{C}\right) \subset L^{2}\left(\Gamma_{C}\right)$. As a result, $P_{\gamma}(\mathbf{u}) \in L^{2}\left(\Gamma_{C}\right)$ and $A_{\gamma}\left(\mathbf{u}, \mathbf{v}^{h}\right)$ makes sense. On the one hand, we use the definition of $P_{\gamma}$, of $A_{\gamma}(\cdot, \cdot)$ and the reformulation (5) of contact conditions to obtain:

$$
\begin{aligned}
& A_{\gamma}\left(\mathbf{u}, \mathbf{v}^{h}\right)+\int_{\Gamma_{C}} \frac{1}{\gamma}\left[P_{\gamma}(\mathbf{u})\right]_{+} P_{\gamma}\left(\mathbf{v}^{h}\right) d \Gamma \\
= & a\left(\mathbf{u}, \mathbf{v}^{h}\right)-\int_{\Gamma_{C}} \gamma \sigma_{n}(\mathbf{u}) \sigma_{n}\left(\mathbf{v}^{h}\right) d \Gamma+\int_{\Gamma_{C}} \frac{1}{\gamma}\left(-\gamma \sigma_{n}(\mathbf{u})\right)\left(v_{n}^{h}-\gamma \sigma_{n}\left(\mathbf{v}^{h}\right)\right) d \Gamma \\
= & a\left(\mathbf{u}, \mathbf{v}^{h}\right)-\int_{\Gamma_{C}} \sigma_{n}(\mathbf{u}) v_{n}^{h} d \Gamma .
\end{aligned}
$$

On the other hand, with equations (1)-(2) and integration by parts, it holds:

$$
a\left(\mathbf{u}, \mathbf{v}^{h}\right)-\int_{\Gamma_{C}} \sigma_{n}(\mathbf{u}) v_{n}^{h} d \Gamma=L\left(\mathbf{v}^{h}\right)
$$

which ends the proof.

\subsection{Well-posedness}

To prove well-posedness of our Nitsche-based formulation, we need first the following classical lemma that we recall here for convenience of the reader.

Lemma 3.2. Let $\gamma_{0}$ be sufficiently small. Then the bilinear form $A_{\gamma}(\cdot, \cdot)$ is elliptic on $\mathbf{V}^{h}$ : there exists $C$, independent of the parameter $\gamma_{0}$ and of the mesh size $h$, such that:

$$
A_{\gamma}\left(\mathbf{v}^{h}, \mathbf{v}^{h}\right) \geq C\left\|\mathbf{v}^{h}\right\|_{1, \Omega}^{2}
$$

for all $\mathbf{v}^{h} \in \mathbf{V}^{h}$.

Proof: Note that we can suppose without loss of generality that $\Gamma_{C}$ is a straight line segment parallel to the $x$-axis. Let $E$ be an edge of a triangle on $\Gamma_{C}$ and let $T \in \mathcal{T}^{h}$ be the element containing $E$. Consequently we deduce, for any $\mathbf{v}^{h} \in \mathbf{V}^{h}$ :

$$
\begin{aligned}
\left\|\sigma_{n}\left(\mathbf{v}^{h}\right)\right\|_{0, E} & =\left\|\sigma_{y y}\left(\mathbf{v}^{h}\right)\right\|_{0, E} \\
& =\frac{|E|^{\frac{1}{2}}}{|T|^{\frac{1}{2}}}\left\|\sigma_{y y}\left(\mathbf{v}^{h}\right)\right\|_{0, T} \\
& \leq C h_{T}^{-\frac{1}{2}}\left\|\sigma_{y y}\left(\mathbf{v}^{h}\right)\right\|_{0, T} \\
& =C\left(\frac{\gamma}{\gamma_{0}}\right)^{-\frac{1}{2}}\left\|\sigma_{y y}\left(\mathbf{v}^{h}\right)\right\|_{0, T}
\end{aligned}
$$


By summation on all the edges $E \subset \Gamma_{C}$ we get

$$
\left\|\gamma^{\frac{1}{2}} \sigma_{n}\left(\mathbf{v}^{h}\right)\right\|_{0, \Gamma_{C}}^{2} \leq C \gamma_{0}\left\|\sigma_{y y}\left(\mathbf{v}^{h}\right)\right\|_{0, \Omega}^{2} \leq C \gamma_{0}\left\|\mathbf{v}^{h}\right\|_{1, \Omega}^{2}
$$

Hence, from Korn inequality and (11), when $\gamma_{0}$ is small enough, there exists $C>0$ such that for any $\mathbf{v}^{h} \in \mathbf{V}^{h}$ :

$$
a\left(\mathbf{v}^{h}, \mathbf{v}^{h}\right)-\int_{\Gamma_{C}} \gamma\left(\sigma_{n}\left(\mathbf{v}^{h}\right)\right)^{2} d \Gamma \geq C\left\|\mathbf{v}^{h}\right\|_{1, \Omega}^{2}
$$

We then show that Problem (8) is well-posed using an argument proposed by Brezis for M type and pseudo-monotone operators [9] (see also [30] and [28]):

Theorem 3.3. Suppose that $\gamma_{0}$ is sufficiently small. Then the problem (8) admits one unique solution $\mathbf{u}^{h}$ in $\mathbf{V}^{h}$.

Proof: Using the Riesz representation theorem, we define a (nonlinear) operator $\mathbf{B}^{h}: \mathbf{V}^{h} \rightarrow \mathbf{V}^{h}$, with help of the formula:

$$
\left(\mathbf{B}^{h} \mathbf{v}^{h}, \mathbf{w}^{h}\right)_{1, \Omega}=A_{\gamma}\left(\mathbf{v}^{h}, \mathbf{w}^{h}\right)+\int_{\Gamma_{C}} \frac{1}{\gamma}\left[P_{\gamma}\left(\mathbf{v}^{h}\right)\right]_{+} P_{\gamma}\left(\mathbf{w}^{h}\right) d \Gamma,
$$

for all $\mathbf{v}^{h}, \mathbf{w}^{h} \in \mathbf{V}^{h}$, and where $(\cdot, \cdot)_{1, \Omega}$ stands for the scalar product in $\left(H^{1}(\Omega)\right)^{2}$. Note that Problem (8) is well-posed if and only if $\mathbf{B}$ is a one-to-one operator.

Due to the properties (4), we observe that, for all $a, b \in \mathbb{R}$ :

$$
\begin{aligned}
\left([a]_{+}-[b]_{+}\right)(a-b) & =a[a]_{+}+b[b]_{+}-b[a]_{+}-a[b]_{+} \\
& \geq[a]_{+}^{2}+[b]_{+}^{2}-2[a]_{+}[b]_{+} \\
& =\left([a]_{+}-[b]_{+}\right)^{2} \geq 0 .
\end{aligned}
$$

Let $\mathbf{v}^{h}, \mathbf{w}^{h} \in \mathbf{V}^{h}$, using the above property and then Lemma 3.2, it holds:

$$
\begin{aligned}
& \left(\mathbf{B}^{h} \mathbf{v}^{h}-\mathbf{B}^{h} \mathbf{w}^{h}, \mathbf{v}^{h}-\mathbf{w}^{h}\right)_{1, \Omega} \\
= & A_{\gamma}\left(\mathbf{v}^{h}-\mathbf{w}^{h}, \mathbf{v}^{h}-\mathbf{w}^{h}\right)+\int_{\Gamma_{C}} \frac{1}{\gamma}\left(\left[P_{\gamma}\left(\mathbf{v}^{h}\right)\right]_{+}-\left[P_{\gamma}\left(\mathbf{w}^{h}\right)\right]_{+}\right) P_{\gamma}\left(\mathbf{v}^{h}-\mathbf{w}^{h}\right) d \Gamma \\
\geq & A_{\gamma}\left(\mathbf{v}^{h}-\mathbf{w}^{h}, \mathbf{v}^{h}-\mathbf{w}^{h}\right) \\
\geq & C\left\|\mathbf{v}^{h}-\mathbf{w}^{h}\right\|_{1, \Omega}^{2} .
\end{aligned}
$$

Next, let us show that $\mathbf{B}^{h}$ is also hemicontinuous. Since $\mathbf{V}^{h}$ is a vector space, it is sufficient to show that

$$
[0,1] \ni t \mapsto \varphi(t)=\left(\mathbf{B}^{h}\left(\mathbf{v}^{h}-t \mathbf{w}^{h}\right), \mathbf{w}^{h}\right)_{1, \Omega} \in \mathbb{R}
$$

is a continuous real function, for all $\mathbf{v}^{h}, \mathbf{w}^{h} \in \mathbf{V}^{h}$. Let $s, t \in[0,1]$, we bound: 


$$
\begin{aligned}
& |\varphi(t)-\varphi(s)| \\
= & \left|\left(\mathbf{B}^{h}\left(\mathbf{v}^{h}-t \mathbf{w}^{h}\right)-\mathbf{B}^{h}\left(\mathbf{v}^{h}-s \mathbf{w}^{h}\right), \mathbf{w}^{h}\right)_{1, \Omega}\right| \\
= & \left|A_{\gamma}\left((s-t) \mathbf{w}^{h}, \mathbf{w}^{h}\right)+\int_{\Gamma_{C}} \frac{1}{\gamma}\left(\left[P_{\gamma}\left(\mathbf{v}^{h}-t \mathbf{w}^{h}\right)\right]_{+}-\left[P_{\gamma}\left(\mathbf{v}^{h}-s \mathbf{w}^{h}\right)\right]_{+}\right) P_{\gamma}\left(\mathbf{w}^{h}\right) d \Gamma\right| \\
\leq & |s-t| A_{\gamma}\left(\mathbf{w}^{h}, \mathbf{w}^{h}\right)+\int_{\Gamma_{C}} \frac{1}{\gamma}\left|\left[P_{\gamma}\left(\mathbf{v}^{h}-t \mathbf{w}^{h}\right)\right]_{+}-\left[P_{\gamma}\left(\mathbf{v}^{h}-s \mathbf{w}^{h}\right)\right]_{+}\right|\left|P_{\gamma}\left(\mathbf{w}^{h}\right)\right| d \Gamma .
\end{aligned}
$$

With help of the bound $\left|[a]_{+}-[b]_{+}\right| \leq|a-b|$, for all $a, b \in \mathbb{R}$, and using the linearity of $P_{\gamma}$, we then remark that:

$$
\begin{aligned}
& \int_{\Gamma_{C}} \frac{1}{\gamma}\left|\left[P_{\gamma}\left(\mathbf{v}^{h}-t \mathbf{w}^{h}\right)\right]_{+}-\left[P_{\gamma}\left(\mathbf{v}^{h}-s \mathbf{w}^{h}\right)\right]_{+}\right|\left|P_{\gamma}\left(\mathbf{w}^{h}\right)\right| d \Gamma \\
\leq & \int_{\Gamma_{C}} \frac{1}{\gamma}\left|P_{\gamma}\left(\mathbf{v}^{h}-t \mathbf{w}^{h}\right)-P_{\gamma}\left(\mathbf{v}^{h}-s \mathbf{w}^{h}\right)\right|\left|P_{\gamma}\left(\mathbf{w}^{h}\right)\right| d \Gamma \\
= & \int_{\Gamma_{C}} \frac{1}{\gamma}\left|(s-t) P_{\gamma}\left(\mathbf{w}^{h}\right)\right|\left|P_{\gamma}\left(\mathbf{w}^{h}\right)\right| d \Gamma \\
= & |s-t| \int_{\Gamma_{C}} \frac{1}{\gamma}\left(P_{\gamma}\left(\mathbf{w}^{h}\right)\right)^{2} d \Gamma .
\end{aligned}
$$

It results that:

$$
|\varphi(t)-\varphi(s)| \leq|s-t|\left(A_{\gamma}\left(\mathbf{w}^{h}, \mathbf{w}^{h}\right)+\left\|\gamma^{-\frac{1}{2}} P_{\gamma}\left(\mathbf{w}^{h}\right)\right\|_{0, \Gamma_{C}}^{2}\right),
$$

which means that $\varphi$ is Lipschitz, so that $\mathbf{B}^{h}$ is hemicontinuous. Since property (12) also holds, we finally apply the Corollary 15 (p.126) of [9] to conclude that $\mathbf{B}^{h}$ is a one-to-one operator. This ends the proof.

\subsection{Error analysis}

Our Nitsche-based method (8) converges optimally when the mesh parameter $h$ vanishes. This is the object of the following theorems. First, we establish an abstract error estimate.

Theorem 3.4. Suppose that the solution $\mathbf{u}$ of Problem (3) belongs to $\left(H^{\frac{3}{2}+\nu}(\Omega)\right)^{2}$ with $\nu>0$. Suppose also that the parameter $\gamma_{0}$ is sufficiently small. The solution $\mathbf{u}^{h}$ of Problem (8) satisfies the following error estimate:

$$
\begin{gathered}
\left\|\mathbf{u}-\mathbf{u}^{h}\right\|_{1, \Omega}+\left\|\gamma^{\frac{1}{2}}\left(\sigma_{n}(\mathbf{u})+\frac{1}{\gamma}\left[P_{\gamma}\left(\mathbf{u}^{h}\right)\right]_{+}\right)\right\|_{0, \Gamma_{C}} \\
\leq \quad C \inf _{\mathbf{v}^{h} \in \mathbf{V}^{h}}\left(\left(\left\|\mathbf{u}-\mathbf{v}^{h}\right\|_{1, \Omega}+\left\|\gamma^{-\frac{1}{2}}\left(u_{n}-v_{n}^{h}\right)\right\|_{0, \Gamma_{C}}+\left\|\gamma^{\frac{1}{2}} \sigma_{n}\left(\mathbf{u}-\mathbf{v}^{h}\right)\right\|_{0, \Gamma_{C}}\right),\right.
\end{gathered}
$$

with $C>0$ a constant, independent of $h$ and $\mathbf{u}$. 
Proof: Let $\mathbf{v}^{h} \in \mathbf{V}^{h}$. We first use the $\mathbf{V}$-ellipticity and the continuity of $a(\cdot, \cdot)$, as well as Young's inequality, to obtain:

$$
\begin{aligned}
\alpha\left\|\mathbf{u}-\mathbf{u}^{h}\right\|_{1, \Omega}^{2} & \leq a\left(\mathbf{u}-\mathbf{u}^{h}, \mathbf{u}-\mathbf{u}^{h}\right) \\
& =a\left(\mathbf{u}-\mathbf{u}^{h},\left(\mathbf{u}-\mathbf{v}^{h}\right)+\left(\mathbf{v}^{h}-\mathbf{u}^{h}\right)\right) \\
& \leq C\left\|\mathbf{u}-\mathbf{u}^{h}\right\|_{1, \Omega}\left\|\mathbf{u}-\mathbf{v}^{h}\right\|_{1, \Omega}+a\left(\mathbf{u}-\mathbf{u}^{h}, \mathbf{v}^{h}-\mathbf{u}^{h}\right) \\
& \leq \frac{\alpha}{2}\left\|\mathbf{u}-\mathbf{u}^{h}\right\|_{1, \Omega}^{2}+\frac{C^{2}}{2 \alpha}\left\|\mathbf{u}-\mathbf{v}^{h}\right\|_{1, \Omega}^{2}+a\left(\mathbf{u}, \mathbf{v}^{h}-\mathbf{u}^{h}\right)-a\left(\mathbf{u}^{h}, \mathbf{v}^{h}-\mathbf{u}^{h}\right),
\end{aligned}
$$

with $\alpha>0$ the ellipticity constant. We can transform the term $a\left(\mathbf{u}, \mathbf{v}^{h}-\mathbf{u}^{h}\right)-a\left(\mathbf{u}^{h}, \mathbf{v}^{h}-\mathbf{u}^{h}\right)$ since $\mathbf{u}$ solves (3) and $\mathbf{u}^{h}$ solves (8), which yields:

$$
\begin{aligned}
\frac{\alpha}{2}\left\|\mathbf{u}-\mathbf{u}^{h}\right\|_{1, \Omega}^{2} \leq & \frac{C^{2}}{2 \alpha}\left\|\mathbf{u}-\mathbf{v}^{h}\right\|_{1, \Omega}^{2}+\int_{\Gamma_{C}} \sigma_{n}(\mathbf{u})\left(v_{n}^{h}-u_{n}^{h}\right) d \Gamma \\
& -\int_{\Gamma_{C}} \gamma \sigma_{n}\left(\mathbf{u}^{h}\right) \sigma_{n}\left(\mathbf{v}^{h}-\mathbf{u}^{h}\right) d \Gamma+\int_{\Gamma_{C}} \frac{1}{\gamma}\left[P_{\gamma}\left(\mathbf{u}^{h}\right)\right]_{+} P_{\gamma}\left(\mathbf{v}^{h}-\mathbf{u}^{h}\right) d \Gamma \\
& =\frac{C^{2}}{2 \alpha}\left\|\mathbf{u}-\mathbf{v}^{h}\right\|_{1, \Omega}^{2}+\int_{\Gamma_{C}} \sigma_{n}(\mathbf{u})\left(v_{n}^{h}-u_{n}^{h}\right) d \Gamma \\
& -\int_{\Gamma_{C}} \gamma \sigma_{n}\left(\mathbf{u}^{h}\right) \sigma_{n}\left(\mathbf{v}^{h}-\mathbf{u}^{h}\right) d \Gamma+\int_{\Gamma_{C}} \frac{1}{\gamma}\left[P_{\gamma}\left(\mathbf{u}^{h}\right)\right]_{+}\left(v_{n}^{h}-\gamma \sigma_{n}\left(\mathbf{v}^{h}\right)\right) d \Gamma \\
& -\int_{\Gamma_{C}} \frac{1}{\gamma}\left[P_{\gamma}\left(\mathbf{u}^{h}\right)\right]_{+}^{2} d \Gamma,
\end{aligned}
$$

where we use (4). We now write

$$
\begin{aligned}
& \int_{\Gamma_{C}} \sigma_{n}(\mathbf{u})\left(v_{n}^{h}-u_{n}^{h}\right) d \Gamma+\int_{\Gamma_{C}} \frac{1}{\gamma}\left[P_{\gamma}\left(\mathbf{u}^{h}\right)\right]_{+} v_{n}^{h} d \Gamma \\
= & \int_{\Gamma_{C}}\left(\sigma_{n}(\mathbf{u})+\frac{1}{\gamma}\left[P_{\gamma}\left(\mathbf{u}^{h}\right)\right]_{+}\right)\left(v_{n}^{h}-u_{n}\right) d \Gamma+\int_{\Gamma_{C}} \sigma_{n}(\mathbf{u})\left(u_{n}-u_{n}^{h}\right) d \Gamma+\int_{\Gamma_{C}} \frac{1}{\gamma}\left[P_{\gamma}\left(\mathbf{u}^{h}\right)\right]_{+} u_{n} d \Gamma \\
\leq & \int_{\Gamma_{C}}\left(\sigma_{n}(\mathbf{u})+\frac{1}{\gamma}\left[P_{\gamma}\left(\mathbf{u}^{h}\right)\right]_{+}\right)\left(v_{n}^{h}-u_{n}\right) d \Gamma+\int_{\Gamma_{C}} \sigma_{n}(\mathbf{u})\left(u_{n}-u_{n}^{h}\right) d \Gamma,
\end{aligned}
$$

because of contact condition (2)-(i). We use this last inequality into the global estimate (14) to obtain: 


$$
\begin{aligned}
& \frac{\alpha}{2}\left\|\mathbf{u}-\mathbf{u}^{h}\right\|_{1, \Omega}^{2} \\
\leq & \frac{C^{2}}{2 \alpha}\left\|\mathbf{u}-\mathbf{v}^{h}\right\|_{1, \Omega}^{2}+\int_{\Gamma_{C}}\left(\sigma_{n}(\mathbf{u})+\frac{1}{\gamma}\left[P_{\gamma}\left(\mathbf{u}^{h}\right)\right]_{+}\right)\left(v_{n}^{h}-u_{n}\right) d \Gamma+\int_{\Gamma_{C}} \sigma_{n}(\mathbf{u})\left(u_{n}-u_{n}^{h}\right) d \Gamma \\
& -\int_{\Gamma_{C}} \gamma \sigma_{n}\left(\mathbf{u}^{h}\right) \sigma_{n}\left(\mathbf{v}^{h}-\mathbf{u}^{h}\right) d \Gamma-\int_{\Gamma_{C}} \frac{1}{\gamma}\left[P_{\gamma}\left(\mathbf{u}^{h}\right)\right]_{+} \gamma \sigma_{n}\left(\mathbf{v}^{h}\right) d \Gamma-\int_{\Gamma_{C}} \frac{1}{\gamma}\left[P_{\gamma}\left(\mathbf{u}^{h}\right)\right]_{+}^{2} d \Gamma \\
= & \frac{C^{2}}{2 \alpha}\left\|\mathbf{u}-\mathbf{v}^{h}\right\|_{1, \Omega}^{2}+\int_{\Gamma_{C}}\left(\sigma_{n}(\mathbf{u})+\frac{1}{\gamma}\left[P_{\gamma}\left(\mathbf{u}^{h}\right)\right]_{+}\right)\left(v_{n}^{h}-u_{n}\right) d \Gamma+\mathcal{T} .
\end{aligned}
$$

The integral term in (15) is bounded thanks to Cauchy-Schwarz and Young inequalities:

$$
\begin{aligned}
& \int_{\Gamma_{C}}\left(\sigma_{n}(\mathbf{u})+\frac{1}{\gamma}\left[P_{\gamma}\left(\mathbf{u}^{h}\right)\right]_{+}\right)\left(v_{n}^{h}-u_{n}\right) d \Gamma \\
& \leq\left\|\gamma^{\frac{1}{2}}\left(\sigma_{n}(\mathbf{u})+\frac{1}{\gamma}\left[P_{\gamma}\left(\mathbf{u}^{h}\right)\right]_{+}\right)\right\|_{0, \Gamma_{C}}\left\|\gamma^{-\frac{1}{2}}\left(v_{n}^{h}-u_{n}\right)\right\|_{0, \Gamma_{C}} \\
& \leq \frac{1}{2 \beta_{1}}\left\|\gamma^{\frac{1}{2}}\left(\sigma_{n}(\mathbf{u})+\frac{1}{\gamma}\left[P_{\gamma}\left(\mathbf{u}^{h}\right)\right]_{+}\right)\right\|_{0, \Gamma_{C}}^{2}+\frac{\beta_{1}}{2}\left\|\gamma^{-\frac{1}{2}}\left(v_{n}^{h}-u_{n}\right)\right\|_{0, \Gamma_{C}}^{2},
\end{aligned}
$$

with $\beta_{1}>0$. We then estimate the (four) integrals in (15) coming from $\mathcal{T}$. Using (2)-(iii), we get

$$
\begin{aligned}
\mathcal{T}= & -\int_{\Gamma_{C}} \sigma_{n}(\mathbf{u}) u_{n}^{h} d \Gamma-\int_{\Gamma_{C}} \gamma \sigma_{n}\left(\mathbf{u}^{h}\right) \sigma_{n}\left(\mathbf{v}^{h}-\mathbf{u}^{h}\right) d \Gamma-\int_{\Gamma_{C}} \frac{1}{\gamma}\left[P_{\gamma}\left(\mathbf{u}^{h}\right)\right]_{+} \gamma \sigma_{n}\left(\mathbf{v}^{h}\right) d \Gamma \\
& -\int_{\Gamma_{C}} \frac{1}{\gamma}\left[P_{\gamma}\left(\mathbf{u}^{h}\right)\right]_{+}^{2} d \Gamma \\
= & -\int_{\Gamma_{C}} \sigma_{n}(\mathbf{u})\left(u_{n}^{h}-\gamma \sigma_{n}\left(\mathbf{u}^{h}\right)\right) d \Gamma-\int_{\Gamma_{C}} \gamma \sigma_{n}(\mathbf{u}) \sigma_{n}\left(\mathbf{u}^{h}\right) d \Gamma \\
& -\int_{\Gamma_{C}} \gamma \sigma_{n}\left(\mathbf{u}^{h}\right) \sigma_{n}\left(\mathbf{v}^{h}-\mathbf{u}^{h}\right) d \Gamma-\int_{\Gamma_{C}} \frac{1}{\gamma}\left[P_{\gamma}\left(\mathbf{u}^{h}\right)\right]_{+} \gamma \sigma_{n}\left(\mathbf{v}^{h}\right) d \Gamma-\int_{\Gamma_{C}} \frac{1}{\gamma}\left[P_{\gamma}\left(\mathbf{u}^{h}\right)\right]_{+}^{2} d \Gamma .
\end{aligned}
$$

Once again we use (4) and contact condition (2)-(ii) to note that:

$$
-\int_{\Gamma_{C}} \sigma_{n}(\mathbf{u})\left(u_{n}^{h}-\gamma \sigma_{n}\left(\mathbf{u}^{h}\right)\right) d \Gamma=-\int_{\Gamma_{C}} \sigma_{n}(\mathbf{u}) P_{\gamma}\left(\mathbf{u}^{h}\right) d \Gamma \leq-\int_{\Gamma_{C}} \sigma_{n}(\mathbf{u})\left[P_{\gamma}\left(\mathbf{u}^{h}\right)\right]_{+} d \Gamma .
$$

It results into: 


$$
\begin{aligned}
\mathcal{T} \leq & -\int_{\Gamma_{C}} \sigma_{n}(\mathbf{u})\left[P_{\gamma}\left(\mathbf{u}^{h}\right)\right]_{+} d \Gamma-\int_{\Gamma_{C}} \gamma \sigma_{n}(\mathbf{u}) \sigma_{n}\left(\mathbf{u}^{h}\right) d \Gamma \\
& -\int_{\Gamma_{C}} \gamma \sigma_{n}\left(\mathbf{u}^{h}\right) \sigma_{n}\left(\mathbf{v}^{h}-\mathbf{u}^{h}\right) d \Gamma-\int_{\Gamma_{C}} \frac{1}{\gamma}\left[P_{\gamma}\left(\mathbf{u}^{h}\right)\right]_{+} \gamma \sigma_{n}\left(\mathbf{v}^{h}\right) d \Gamma-\int_{\Gamma_{C}} \frac{1}{\gamma}\left[P_{\gamma}\left(\mathbf{u}^{h}\right)\right]_{+}^{2} d \Gamma \\
= & -\int_{\Gamma_{C}}\left(\sigma_{n}(\mathbf{u})+\frac{1}{\gamma}\left[P_{\gamma}\left(\mathbf{u}^{h}\right)\right]_{+}\right)\left[P_{\gamma}\left(\mathbf{u}^{h}\right)\right]_{+} d \Gamma-\int_{\Gamma_{C}} \gamma \sigma_{n}(\mathbf{u}) \sigma_{n}\left(\mathbf{u}^{h}\right) d \Gamma \\
& -\int_{\Gamma_{C}} \gamma \sigma_{n}\left(\mathbf{u}^{h}\right) \sigma_{n}\left(\mathbf{v}^{h}-\mathbf{u}^{h}\right) d \Gamma-\int_{\Gamma_{C}}\left(\frac{1}{\gamma}\left[P_{\gamma}\left(\mathbf{u}^{h}\right)\right]_{+}+\sigma_{n}(\mathbf{u})\right) \gamma \sigma_{n}\left(\mathbf{v}^{h}\right) d \Gamma \\
& +\int_{\Gamma_{C}} \gamma \sigma_{n}(\mathbf{u}) \sigma_{n}\left(\mathbf{v}^{h}\right) d \Gamma \\
= & -\int_{\Gamma_{C}} \gamma\left(\sigma_{n}(\mathbf{u})+\frac{1}{\gamma}\left[P_{\gamma}\left(\mathbf{u}^{h}\right)\right]_{+}\right)\left(\frac{1}{\gamma}\left[P_{\gamma}\left(\mathbf{u}^{h}\right)\right]_{+}+\sigma_{n}(\mathbf{u})\right) d \Gamma-\int_{\Gamma_{C}} \gamma \sigma_{n}(\mathbf{u}) \sigma_{n}\left(\mathbf{u}^{h}\right) d \Gamma \\
& -\int_{\Gamma_{C}} \gamma \sigma_{n}\left(\mathbf{u}^{h}\right) \sigma_{n}\left(\mathbf{v}^{h}-\mathbf{u}^{h}\right) d \Gamma+\int_{\Gamma_{C}}^{\gamma}\left(\sigma_{n}(\mathbf{u})+\frac{1}{\gamma}\left[P_{\gamma}\left(\mathbf{u}^{h}\right)\right]_{+}\right) \sigma_{n}\left(\mathbf{u}-\mathbf{v}^{h}\right) d \Gamma \\
& +\int_{\Gamma_{C}} \gamma \sigma_{n}(\mathbf{u}) \sigma_{n}\left(\mathbf{v}^{h}\right) d \Gamma .
\end{aligned}
$$

As a consequence

$$
\begin{aligned}
\mathcal{T}+\left\|\gamma^{\frac{1}{2}}\left(\sigma_{n}(\mathbf{u})+\frac{1}{\gamma}\left[P_{\gamma}\left(\mathbf{u}^{h}\right)\right]_{+}\right)\right\|_{0, \Gamma_{C}}^{2} \leq & \int_{\Gamma_{C}} \gamma\left(\sigma_{n}(\mathbf{u})+\frac{1}{\gamma}\left[P_{\gamma}\left(\mathbf{u}^{h}\right)\right]_{+}\right) \sigma_{n}\left(\mathbf{u}-\mathbf{v}^{h}\right) d \Gamma \\
& +\int_{\Gamma_{C}} \gamma \sigma_{n}\left(\mathbf{u}-\mathbf{u}^{h}\right) \sigma_{n}\left(\mathbf{v}^{h}-\mathbf{u}^{h}\right) d \Gamma
\end{aligned}
$$

With help of Cauchy-Schwarz and Young inequalities, we bound the first term in (17) :

$$
\begin{aligned}
\int_{\Gamma_{C}} \gamma\left(\sigma_{n}(\mathbf{u})+\frac{1}{\gamma}\left[P_{\gamma}\left(\mathbf{u}^{h}\right)\right]_{+}\right) \sigma_{n}\left(\mathbf{u}-\mathbf{v}^{h}\right) d \Gamma \leq & \frac{1}{2 \beta_{2}}\left\|\gamma^{\frac{1}{2}}\left(\sigma_{n}(\mathbf{u})+\frac{1}{\gamma}\left[P_{\gamma}\left(\mathbf{u}^{h}\right)\right]_{+}\right)\right\|_{0, \Gamma_{C}}^{2} \\
& +\frac{\beta_{2}}{2}\left\|\gamma^{\frac{1}{2}} \sigma_{n}\left(\mathbf{u}-\mathbf{v}^{h}\right)\right\|_{0, \Gamma_{C}}^{2},
\end{aligned}
$$

with $\beta_{2}>0$. 
The second term in (17) is bounded as follows by using (11) and Young inequality:

$$
\begin{aligned}
& \int_{\Gamma_{C}} \gamma \sigma_{n}\left(\mathbf{u}-\mathbf{u}^{h}\right) \sigma_{n}\left(\mathbf{v}^{h}-\mathbf{u}^{h}\right) d \Gamma \\
\leq & \left\|\gamma^{\frac{1}{2}} \sigma_{n}\left(\mathbf{u}-\mathbf{u}^{h}\right)\right\|_{0, \Gamma_{C}}\left\|\gamma^{\frac{1}{2}} \sigma_{n}\left(\mathbf{v}^{h}-\mathbf{u}^{h}\right)\right\|_{0, \Gamma_{C}} \\
\leq & C \gamma_{0}^{\frac{1}{2}}\left\|\mathbf{v}^{h}-\mathbf{u}^{h}\right\|_{1, \Omega}\left(\left\|\gamma^{\frac{1}{2}} \sigma_{n}\left(\mathbf{u}-\mathbf{v}^{h}\right)\right\|_{0, \Gamma_{C}}+\left\|\gamma^{\frac{1}{2}} \sigma_{n}\left(\mathbf{v}^{h}-\mathbf{u}^{h}\right)\right\|_{0, \Gamma_{C}}\right) \\
\leq & C\left(\gamma_{0}\left\|\mathbf{v}^{h}-\mathbf{u}^{h}\right\|_{1, \Omega}^{2}+\left\|\gamma^{\frac{1}{2}} \sigma_{n}\left(\mathbf{u}-\mathbf{v}^{h}\right)\right\|_{0, \Gamma_{C}}^{2}\right) \\
\leq & C\left(\gamma_{0}\left\|\mathbf{u}-\mathbf{u}^{h}\right\|_{1, \Omega}^{2}+\gamma_{0}\left\|\mathbf{u}-\mathbf{v}^{h}\right\|_{1, \Omega}^{2}+\left\|\gamma^{\frac{1}{2}} \sigma_{n}\left(\mathbf{u}-\mathbf{v}^{h}\right)\right\|_{0, \Gamma_{C}}^{2}\right) .
\end{aligned}
$$

Putting together (18) and (19) in (17) gives:

$$
\begin{aligned}
\mathcal{T}+\left(1-\frac{1}{2 \beta_{2}}\right)\left\|\gamma^{\frac{1}{2}}\left(\sigma_{n}(\mathbf{u})+\frac{1}{\gamma}\left[P_{\gamma}\left(\mathbf{u}^{h}\right)\right]_{+}\right)\right\|_{0, \Gamma_{C}}^{2} \leq & C\left(\gamma_{0}\left\|\mathbf{u}-\mathbf{u}^{h}\right\|_{1, \Omega}^{2}+\gamma_{0}\left\|\mathbf{u}-\mathbf{v}^{h}\right\|_{1, \Omega}^{2}\right) \\
& +\left(C+\frac{\beta_{2}}{2}\right)\left\|\gamma^{\frac{1}{2}} \sigma_{n}\left(\mathbf{u}-\mathbf{v}^{h}\right)\right\|_{0, \Gamma_{C}}^{2} .
\end{aligned}
$$

We finally combine estimates (16) and (20) into (15) to get:

$$
\begin{aligned}
& \left(\frac{\alpha}{2}-C \gamma_{0}\right)\left\|\mathbf{u}-\mathbf{u}^{h}\right\|_{1, \Omega}^{2}+\left(1-\frac{1}{2 \beta_{1}}-\frac{1}{2 \beta_{2}}\right)\left\|\gamma^{\frac{1}{2}}\left(\sigma_{n}(\mathbf{u})+\frac{1}{\gamma}\left[P_{\gamma}\left(\mathbf{u}^{h}\right)\right]_{+}\right)\right\|_{0, \Gamma_{C}}^{2} \\
\leq & \left(\frac{C^{2}}{2 \alpha}+C \gamma_{0}\right)\left\|\mathbf{u}-\mathbf{v}^{h}\right\|_{1, \Omega}^{2}+\frac{\beta_{1}}{2}\left\|\gamma^{-\frac{1}{2}}\left(u_{n}-v_{n}^{h}\right)\right\|_{0, \Gamma_{C}}^{2}+\left(C+\frac{\beta_{2}}{2}\right)\left\|\gamma^{\frac{1}{2}} \sigma_{n}\left(\mathbf{u}-\mathbf{v}^{h}\right)\right\|_{0, \Gamma_{C}}^{2} .
\end{aligned}
$$

To conclude, take $\gamma_{0}$ sufficiently small and for instance $\beta_{1}=\beta_{2}=2$.

The optimal convergence of the method is stated below.

Theorem 3.5. Suppose that the solution $\mathbf{u}$ of Problem (3) belongs to $\left(H^{\frac{3}{2}+\nu}(\Omega)\right)^{2}$ with $0<\nu \leq$ 1/2. Suppose also that the parameter $\gamma_{0}$ is sufficiently small. The solution $\mathbf{u}^{h}$ of Problem (8) satisfies the following error estimate:

$$
\left\|\mathbf{u}-\mathbf{u}^{h}\right\|_{1, \Omega}+\left\|\gamma^{\frac{1}{2}}\left(\sigma_{n}(\mathbf{u})+\frac{1}{\gamma}\left[P_{\gamma}\left(\mathbf{u}^{h}\right)\right]_{+}\right)\right\|_{0, \Gamma_{C}} \leq C h^{\frac{1}{2}+\nu}\|\mathbf{u}\|_{\frac{3}{2}+\nu, \Omega},
$$

with $C>0$ a constant, independent of $h$ and $\mathbf{u}$.

Proof: We need to bound the right terms in estimate (13) and we choose $\mathbf{v}^{h}=\mathcal{I}^{h} \mathbf{u}$ where $\mathcal{I}^{h}$ stands for the Lagrange interpolation operator mapping onto $\mathbf{V}^{h}$. The estimation of the Lagrange interpolation error in $H^{1}$-norm on a domain $\Omega$ is classical (see, e.g., $[8,13]$ ):

$$
\left\|\mathbf{u}-\mathcal{I}^{h} \mathbf{u}\right\|_{1, \Omega} \leq C h^{\frac{1}{2}+\nu}\|\mathbf{u}\|_{\frac{3}{2}+\nu, \Omega}
$$


for $-1 / 2<\nu \leq 1 / 2$.

The estimation of the term $\left\|\gamma^{-\frac{1}{2}}\left(u_{n}-\left(\mathcal{I}^{h} \mathbf{u}\right)_{n}\right)\right\|_{0, \Gamma_{C}}$ can be done in a very similar manner to [23]. Indeed, let $E$ be an edge of a triangle $T \in \mathcal{T}^{h}$ on $\Gamma_{C}$ :

$$
\left\|\gamma^{-\frac{1}{2}}\left(u_{n}-\left(\mathcal{I}^{h} \mathbf{u}\right)_{n}\right)\right\|_{0, E} \leq C h_{T}^{-\frac{1}{2}} h_{T}^{1+\nu}\left\|u_{n}\right\|_{1+\nu, E}=C h^{\frac{1}{2}+\nu}\left\|u_{n}\right\|_{1+\nu, E},
$$

(see [13] for instance). By summation on all the edges and the trace theorem, it results:

$$
\left\|\gamma^{-\frac{1}{2}}\left(u_{n}-\left(\mathcal{I}^{h} \mathbf{u}\right)_{n}\right)\right\|_{0, \Gamma_{C}} \leq C h^{\frac{1}{2}+\nu}\left\|u_{n}\right\|_{1+\nu, \Gamma_{C}} \leq C h^{\frac{1}{2}+\nu}\|\mathbf{u}\|_{\frac{3}{2}+\nu, \Omega}
$$

From [16] (see also the details in [15]), the following estimate also holds:

$$
\left\|\gamma^{\frac{1}{2}} \sigma_{n}\left(\mathbf{u}-\mathcal{I}^{h} \mathbf{u}\right)\right\|_{0, \Gamma_{C}} \leq C h^{\frac{1}{2}+\nu}\|\mathbf{u}\|_{\frac{3}{2}+\nu, \Omega} .
$$

We conclude by inserting the three estimates (22)-(24) into (13).

Actually we are not able to obtain estimates for the displacements in the $L^{2}$-norm $\left\|\mathbf{u}-\mathbf{u}^{h}\right\|_{0, \Omega}$ (and also $\left\|\mathbf{u}-\mathbf{u}^{h}\right\|_{0, \Gamma_{C}}$ ) by using the Aubin-Nitsche argument as it is achieved in the linear case (see [16]). Note also that the $L^{2}(\Omega)$-norm estimates for contact problems are not easy to prove and there are to our knowledge only few estimates (see [12]). Nevertheless, we can easily obtain the following error estimate on the weighted $L^{2}\left(\Gamma_{C}\right)$-norm on the normal constraint $\left\|\gamma^{\frac{1}{2}} \sigma_{n}\left(\mathbf{u}-\mathbf{u}_{h}\right)\right\|_{0, \Gamma_{C}}$ (note that $\sigma_{n}\left(\mathbf{u}_{h}\right) \neq-\frac{1}{\gamma}\left[P_{\gamma}\left(\mathbf{u}^{h}\right)\right]_{+}$on $\Gamma_{C}$ contrary to the continuous case).

Corollary 3.6. Suppose that the solution u of Problem (3) belongs to $\left(H^{\frac{3}{2}+\nu}(\Omega)\right)^{2}$ with $0<$ $\nu \leq 1 / 2$. Suppose also that the parameter $\gamma_{0}$ is sufficiently small. The solution $\mathbf{u}^{h}$ of Problem (8) satisfies the following error estimate:

$$
\left\|\gamma^{\frac{1}{2}} \sigma_{n}\left(\mathbf{u}-\mathbf{u}_{h}\right)\right\|_{0, \Gamma_{C}} \leq C h^{\frac{1}{2}+\nu}\|\mathbf{u}\|_{\frac{3}{2}+\nu, \Omega}
$$

with $C>0$ a constant, independent of $h$ and $\mathbf{u}$.

Proof: We use (24), (11), (22) and (21) to establish the bound:

$$
\begin{aligned}
\left\|\gamma^{\frac{1}{2}} \sigma_{n}\left(\mathbf{u}-\mathbf{u}_{h}\right)\right\|_{0, \Gamma_{C}} & \leq\left\|\gamma^{\frac{1}{2}} \sigma_{n}\left(\mathbf{u}-\mathcal{I}^{h} \mathbf{u}\right)\right\|_{0, \Gamma_{C}}+\left\|\gamma^{\frac{1}{2}} \sigma_{n}\left(\mathcal{I}^{h} \mathbf{u}-\mathbf{u}_{h}\right)\right\|_{0, \Gamma_{C}} \\
& \leq C h^{\frac{1}{2}+\nu}\|\mathbf{u}\|_{\frac{3}{2}+\nu, \Omega}+C \gamma_{0}^{\frac{1}{2}}\left(\left\|\mathcal{I}^{h} \mathbf{u}-\mathbf{u}\right\|_{1, \Omega}+\left\|\mathbf{u}-\mathbf{u}_{h}\right\|_{1, \Omega}\right) \\
& \leq C h^{\frac{1}{2}+\nu}\|\mathbf{u}\|_{\frac{3}{2}+\nu, \Omega}
\end{aligned}
$$

Remark 3.7. Although contact problems are known to be limited in regularity (the regularity $H^{5 / 2}(\Omega)$ can not generally be passed beyond for such inequality problems), the use of quadratic finite element methods can be of interest in particular for the most regular solutions lying in $H^{s}(\Omega), 2<s<5 / 2$ (as it is considered in e.g., [5, 22, 32, 37]). It is easy to check that the 
trace inequality (11) is satisfied in the case of quadratic elements so that Theorem 3.4 still holds. Moreover, since estimates (22)-(24) remain true in the case of quadratic finite elements (see [15] and [16] for estimate (24)), we come to the conclusion that the optimal convergence rates (21) and (25) hold for $0<\nu \leq 3 / 2$ when quadratic finite elements are used.

\section{Conclusion and perspectives}

In this paper we propose a new finite element approximation for the unilateral contact problem in two-dimensional linear elasticity and we achieve the corresponding numerical analysis. This analysis allows us to obtain the first optimal a priori error estimate under $H^{s}$ regularity $(3 / 2<$ $s \leq 2$ ) on the solution without any additional assumption. The numerical experiments as well as the extension to the three-dimensional case should be considered in a forthcoming study.

\section{Acknowledgements.}

We thank Yves Renard for helpful discussions and comments.

\section{References}

[1] R. A. Adams, Sobolev spaces, Academic Press [A subsidiary of Harcourt Brace Jovanovich, Publishers], New York-London, 1975. Pure and Applied Mathematics, Vol. 65.

[2] D. Arnold, An interior penalty finite element method with discontinuous elements, SIAM J. Numer. Anal., 19 (1982), pp. 742-760.

[3] H. J. C. Barbosa and T. J. R. Hughes, Circumventing the Babuška-Brezzi condition in mixed finite element approximations of elliptic variational inequalities, Comput. Methods Appl. Mech. Engrg., 97 (1992), pp. 193-210.

[4] R. Becker, P. Hansbo, and R. Stenberg, A finite element method for domain decomposition with non-matching grids, M2AN Math. Model. Numer. Anal., 37 (2003), pp. 209-225.

[5] Z. Belhachmi and F. Ben Belgacem, Quadratic finite element approximation of the Signorini problem, Math. Comp., 72 (2003), pp. 83-104.

[6] F. Ben Belgacem, Numerical simulation of some variational inequalities arisen from unilateral contact problems by the finite element methods, SIAM J. Numer. Anal., 37 (2000), pp. 1198-1216.

[7] F. Ben Belgacem And Y. Renard, Hybrid finite element methods for the Signorini problem, Math. Comp., 72 (2003), pp. 1117-1145.

[8] S.-C. Brenner And L.-R. Scott, The Mathematical Theory of Finite Element Methods, vol. 15 of Texts in Applied Mathematics, Springer-Verlag, New York, 2007. 
[9] H. BREzIS, Équations et inéquations non linéaires dans les espaces vectoriels en dualité, Ann. Inst. Fourier (Grenoble), 18 (1968), pp. 115-175.

[10] F. Chouly and P. Hild, On convergence of the penalty method for unilateral contact problems. http://hal.archives-ouvertes.fr/hal-00688641, Apr. 2012.

[11] P. G. Ciarlet, Handbook of Numerical Analysis (eds. P.G. Ciarlet and J.L. Lions), vol. II, North Holland, 1991, ch. 1. "The finite element method for elliptic problems", pp. 17-352.

[12] P. Coorevits, P. Hild, K. Lhalouani, and T. Sassi, Mixed finite element methods for unilateral problems: convergence analysis and numerical studies, Math. Comp., 71 (2002), pp. 1-25.

[13] A. Ern And J.-L. Guermond, Theory and practice of finite elements, vol. 159 of Applied Mathematical Sciences, Springer-Verlag, New York, 2004.

[14] G. Fichera, Problemi elastostatici con vincoli unilaterali: Il problema di Signorini con ambigue condizioni al contorno, Atti Accad. Naz. Lincei Mem. Cl. Sci. Fis. Mat. Natur. Sez. I (8), 7 (1963/1964), pp. 91-140.

[15] A. Fritz, S. Hüeber, And B. I. Wohlmuth, A comparison of mortar and Nitsche techniques for linear elasticity, Universität Stuttgart, IANS Preprint, (2003).

[16] — A comparison of mortar and Nitsche techniques for linear elasticity, Calcolo, 41 (2004), pp. 115-137.

[17] R. Glowinski and P. Le Tallec, Augmented Lagrangian and operator-splitting methods in nonlinear mechanics, vol. 9 of SIAM Studies in Applied Mathematics, Society for Industrial and Applied Mathematics (SIAM), Philadelphia, PA, 1989.

[18] W. Han And M. Sofonea, Quasistatic contact problems in viscoelasticity and viscoplasticity, vol. 30 of AMS/IP Studies in Advanced Mathematics, American Mathematical Society, Providence, RI, 2002.

[19] J. HASLINGER, Finite element analysis for unilateral problems with obstacles on the boundary, Apl. Mat., 22 (1977), pp. 180-188.

[20] J. Haslinger, I. HlaváČek, and J. NeČas, Handbook of Numerical Analysis (eds. P.G. Ciarlet and J.L. Lions), vol. IV, North Holland, 1996, ch. 2. "Numerical methods for unilateral problems in solid mechanics", pp. 313-385.

[21] P. Heintz and P. Hansbo, Stabilized Lagrange multiplier methods for bilateral elastic contact with friction, Comput. Methods Appl. Mech. Engrg., 195 (2006), pp. 4323-4333.

[22] P. Hild and P. Laborde, Quadratic finite element methods for unilateral contact problems, Appl. Numer. Math., 41 (2002), pp. 401-421. 
[23] P. Hild And Y. Renard, A stabilized Lagrange multiplier method for the finite element approximation of contact problems in elastostatics, Numer. Math., 115 (2010), pp. 101-129.

[24] —_ An improved a priori error analysis for finite element approximations of Signorini's problem, (2012). In revision in SIAM J. Numer. Anal.

[25] S. Hüeber And B. I. Wohlmuth, An optimal a priori error estimate for nonlinear multibody contact problems, SIAM J. Numer. Anal., 43 (2005), pp. 156-173.

[26] T. J. R. Hughes And L. P. Franca, A new finite element formulation for computational fluid dynamics. VII. The Stokes problem with various well-posed boundary conditions: symmetric formulations that converge for all velocity/pressure spaces, Comput. Methods Appl. Mech. Engrg., 65 (1987), pp. 85-96.

[27] N. Kikuchi And J. T. Oden, Contact problems in elasticity: a study of variational inequalities and finite element methods, vol. 8 of SIAM Studies in Applied Mathematics, Society for Industrial and Applied Mathematics (SIAM), Philadelphia, PA, 1988.

[28] N. Kikuchi And Y. J. Song, Penalty-finite-element approximation of a class of unilateral problems in linear elasticity, Quart. Appl. Math., 39 (1981), pp. 1-22.

[29] T. A. Laursen, Computational contact and impact mechanics, Springer-Verlag, Berlin, 2002 .

[30] J.-L. Lions, Quelques méthodes de résolution des problèmes aux limites non linéaires, Dunod, Paris, 1969.

[31] J. Nitsche, Über ein Variationsprinzip zur Lösung von Dirichlet-Problemen bei Verwendung von Teilräumen, die keinen Randbedingungen unterworfen sind, Abhandlungen aus dem Mathematischen Seminar der Universität Hamburg, 36 (1971), pp. 9-15.

[32] A. Popp, B. I. Wohlmuth, M. W. Gee, And W. A. Wall, Dual quadratic mortar finite element methods for $3 D$ finite deformation contact, SIAM J. Sci. Comput., (2012).

[33] Y. RenARD, A quasi-optimal a priori error estimate for the two-dimensional Signorini problem approximated by linear finite elements, Comptes Rendus Mathematique, 350 (2012), pp. 325-328.

[34] F. SCARPINI AND M.-A. VIVALDI, Error estimates for the approximation of some unilateral problems, RAIRO Anal. Numér., 11 (1977), pp. 197-208.

[35] R. Stenberg, On some techniques for approximating boundary conditions in the finite element method, Journal of Computational and Applied Mathematics, 63 (1995), pp. 139148. International Symposium on Mathematical Modelling and Computational Methods Modelling 94 (Prague, 1994). 
[36] B. I. Wohlmuth, Variationally consistent discretization schemes and numerical algorithms for contact problems, Acta Numer., 20 (2011), pp. 569-734.

[37] B. I. Wohlmuth, A. Popp, M. W. Gee, And W. A. Wall, An abstract framework for a priori estimates for contact problems in $3 D$ with quadratic finite elements, Comput. Mech., 49 (2012), pp. 735-747.

[38] P. Wriggers, Computational Contact Mechanics, Wiley, 2002. 